\title{
TE AROHA
}

\author{
Aashika Rajesh, Apoorva.S, Harshini.G, Keerthana Yadunath, Shruti B M* \\ Department of Computer Science and Engineering, GSSS Institute of Engineering and \\ Technology for Women, Mysore, India
}

DOI: https://doi.org/10.21467/proceedings.1.30

* Corresponding author email: shruthibm@gsss.edu.in

\begin{abstract}
This App allows you to donate funds, clothes, books, stationery, etc to appeals and charities directly from your mobile phone, anytime, anywhere. Your friends and colleagues are even notified of your donations through Facebook and Twitter. Charities sorted into categories to make giving even easier. Easily see featured causes. Receipt delivered to your phone and your email address.
\end{abstract}

\section{INTRODUCTION}

The world is moving towards smart phones in this era. Most people prefer the mobility and compactness of a smart phone. Because of this, more and more developers have moved towards making mobile apps. The whole psychology behind this is the idea of convenience. Its way easier to do something over a smart phone than to boot up a laptop/desktop, wait for it to start up, open the app, and so on. Now, coming to the NGO field, there aren't many apps that let you interact with orphanages, NGO s etc. We need to find a way to create a link between a donator and an orphanage so that the information relating to the orphanage can easily be accessed to the donator. As of now, the only way you get to gain more information about them is to actually visit the location. This creates way more steps than necessary and prevents users from actually donating.

\section{LITERATURE SURVEY}

\subsection{Mobile Application Web Service Performance Analysis}

Mobile devices are growing on a pace without precedents. It is estimated that by 2013 there will be about 1.1 Billion Smart phones on use. This quick growth on Smartphone numbers and the appearance of marketplaces where mobile applications can easily be bought or downloaded is turning mobile development into a big market. As the number of users grows in a fast pace, so does the demand for new applications, leading to faster development cycles in order to produce faster results. In many cases, mobile applications are connected to preexisting systems and it is necessary to find a quick, but stable, secure and light way to establish a communication between the mobile application and the pre-existing background system.

(C) 2018 Copyright held by the author(s). Published by AIJR Publisher in Proceedings of the $3^{\text {rd }}$ National Conference on Image Processing, Computing, Communication, Networking and Data Analytics (NCICCNDA 2018), April 28, 2018.

This is an open access article under Creative Commons Attribution-NonCommercial 4.0 International (CC BY-NC 4.0) license, which permits any non-commercial use, distribution, adaptation, and reproduction in any medium, as long as the

AijR license, which permits any non-commercial use, distribution, 
This article aims to show how restful Web services, combined with JSON, may help developers to fill this gap while keeping security, stability and speed.

\subsection{Information System Based on College Campus}

Android mobile apps is used to provide information regarding their college. Android is the fastest growing open source mobile device platform, which in turn is powered by Linux operating system. Android offers a simple yet powerful application development framework (C++/Java and XML) and open access to APIs to build richer mobile applications. Android is a software stack for mobile devices that includes an operating system, middleware and key applications. Today in college student details are entered manually. The student separate records are tedious task. Referring to all these records and updating is needed. There is a chance for more manual errors. As mobile devices have become popular; there appears a new trend to release all kinds of campus information by intelligent mobile terminals. We describe a network for distributing campus information among lecturers and students. The concept of developing campus information system is to ensure that student can access information at any time, at any locations and ad-hoc basic. Information System helps the students and lecturers on campus to find and access information based on ad-hoc basic, which is of interest and relevant to students or lecturers through a smart phone.

\subsection{Performance Analysis of SOAP and Restful Mobile Web Services in Cloud Environment}

Web service can be built using two disparate ways: Standard SOAP based Web Services and Restful Web service. Paper focuses on developing both Web Services with different parameters. We use SOAP, REST API + Jersey API for implementing XML based and Restful Web Services respectively. We have conducted different experiments on both and tested on Apache Tomcat server, Android Emulators well as on Cloud server Google App Engine. Result of experiment shows Restful web services are better in performance than SOAP Web Services.

\subsection{Computers and Electronics in Agriculture}

The relentlessly increasing importance and application of Information and Communication Technologies (ICTs) in Agriculture have given birth to a new field called e-Agriculture, which focus on improving agricultural and rural development through a variety of technologies. In this sense, Agricultural Information Systems (AISs) are distributed sources of information that exploit ICTs to make agricultural processes and decision making more efficient. In order to integrate AISs and therefore build added value AISs, Web Service technologies seem to be the right path towards heterogeneous systems integration. However, there is still uncertain which is the best implementation approach to integrate Web Service-enabled AISs and mobile devices that is the remote information acessors by excellence in rural areas. We comparatively explore the outcomes of employing either Simple Object Access Protocol (SOAP) or

Proceedings of the $3^{\text {rd }}$ National Conference on Image Processing, Computing, Communication, Networking and Data Analytics (NCICCNDA 2018) 

simulator accessed from Android-powered smart phones. Memory usage was 24\% lower in SOAP, but even older and lower-end smart phones have enough RAM to avoid detrimental effects on performance. REST-based approaches broadly incur in less byte transferred compared to SOAP, which has huge implications on costs. That is particularly important when the Internet is accessed via GPRS or $3 \mathrm{G}$ protocols and pay-per-byte data plans as in most of Latin America rural areas. However, when unlimited data usage became less costly and more available in such areas, SOAP might be preferred due to the higher maturity of both the protocol and the available developer environments.

\section{TECHNOLOGY}

3.1 Android: It is a mobile OS developed by google based on the modified version of linux kernel open source software and design primarily for touch screen mobile services.

3.2 Mysql: It is a relational database management systems. It is central component of the LAMP open source web application software stack.

3.3 ISON(Java Script object Notation): In android app the regular text is to converted to converted to JSON format. JSON is an open standard file format that uses human readable text to transmit data objects consisting of attribute value pairs and array data types.

3.4 PHP: It is a general purpose scripting language especially suited for to server side web development it acts primarily acts as a filter taking input from a file or stream containing text or php instructions and outputting another stream of data.

3.5 Java: Java is a general purpose computer programming language that is concurrent, class based, object oriented and specifically designed to have as few implementation dependencies as possible. $\mathrm{I} t$ is intended to let application developers write once and run anywhere.

3.6 Bootstrap: It is a free and open source front end library for designing web sites and web applications. It contains $\mathrm{html}$ and CSS based design and templates for typography, forms, buttons, navigation and other interface components, as well as optional java script extensions.

3.7 HTML: HTML is standard markup language for creating web pages and web applications. With CSS and java script, it forms a traid of cornerstone technologies for world wide web.

3.8 CSS: CSS is a style sheet language used for describing the presentation of a document written in markup language like html. CSS is designed to enable the separation of presentation and content, including layouts, colors, fonts.

\section{HIGH LEVEL DESIGN}

A data flow diagram (DFD) maps out the flow of information for any process or system. If the user is a donor, a screen where the donor can set up an event is shown. The user can set 
the type of donation like food, clothes, stationary, etc, and choose a pickup location. The user also selects a date for the pickup or food distribution.

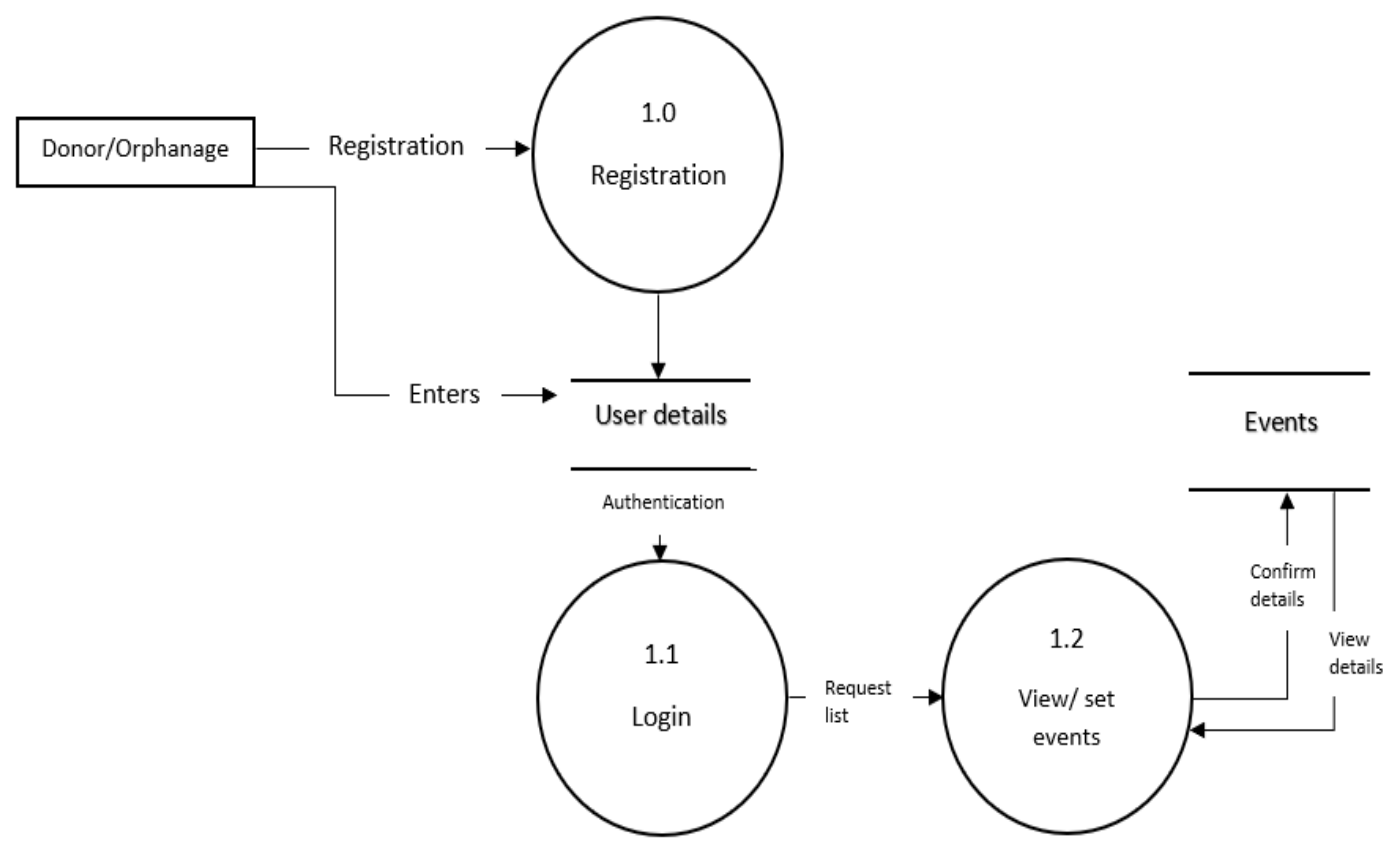

\section{CONCLUSIONS}

By making it easier for an individual to search for and contact NGOs, we aim to not only create awareness about the variety of NGOs out there, but also make the entire process of approaching an NGO more accessible for the normal user. We do so by allowing a potential donator to find NGOs based on his/her needs and set up an event, be it donations in the form of books and stationeries, toys, etc, or cash. For a more sensitive matter such as adoption, the app can provide the contact details of the NGO so that the user can communicate with the concerned individual in person and set things up. Over time, we hope the concept behind Te Aroha becomes more widespread and adopted, thereby aiding in the sustainability of NGOs.

\section{References}

[1] Shilpa Bilawane, Pranali Jambhulkar, "Information System Based On College Campus", Volume 4 Issue 3 March 2015, Page No. 10852-10855

[2] Carlos Rodrigues, José Afonso, and Paulo Tomé," Mobile Application Webservice Performance Analysis: Restful Services with JSON and XML”, Part II, CCIS 220, pp. 162-169, 2011.

Proceedings of the $3^{\text {rd }}$ National Conference on Image Processing, Computing, Communication, Networking and Data Analytics (NCICCNDA 2018) 